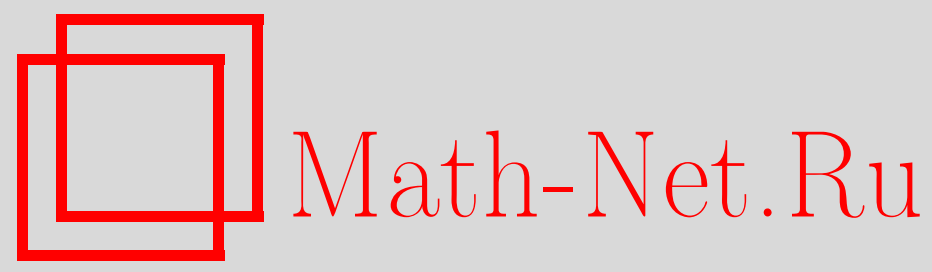

А. П. Шашкин, Максимальное неравенство для слабо зависимого случайного поля, Матем. заметки, 2004, том 75, выпуск 5, 773-782

DOI: https://doi.org/10.4213/mzm70

Использование Общероссийского математического портала Math-Net.Ru подразумевает, что вы прочитали и согласны с пользовательским соглашением http://www.mathnet.ru/rus/agreement

Параметры загрузки:

IP: 54.84 .234 .179

26 апреля 2023 г., 16:31:01

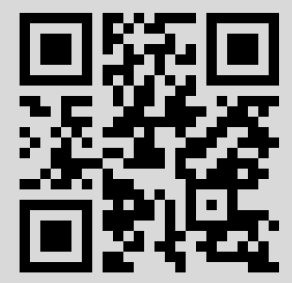




\title{
МАКСИМАЛЬНОЕ НЕРАВЕНСТВО ДЛЯ СЛАБО ЗАВИСИМОГО СЛУЧАЙНОГО ПОЛЯ
}

\section{А. П. Шашкин}

\begin{abstract}
Получено максимальное неравенство для случайного поля, обладающего свойством слабой зависимости, связанным с убыванием ковариаций функций определенного класса от элементов поля, когда расстояние между индексирующими множествами стремится к бесконечности.

Библиографоия: 15 названий.
\end{abstract}

Получение оценок для моментов максимума сумм независимых или определенным образом зависимых случайных величин - классическая задача теории вероятностей (см., например, $[1$, гл. 3], [2, §1.4.3]). При этом рассматриваются как максимумы частных сумм последовательности случайных величин, так и максимумы сумм значений случайных полей, заданных на решетке $\mathbb{Z}^{d}$, берущихся по целочисленным параллелепипедам из некоторого подмножества этой решетки (см. [3]-[5]). Такого рода неравенства, наряду с результатами об асимптотической нормальности, позволяют получать принцип инвариантности и функциональньй закон повторного логарифма (см., например, [3], [5], $[6$, гл. $2, \S 8],[7])$.

Цель данной работы - вьвести максимальное неравенство для класса случайных полей, обладающих свойством зависимости, введенным в [8] (сходные условия зависимости рассматривались также в [9] и [10]). Это свойство заключается в должном убывании ковариаций ограниченных липшицевых функций от непересекающихся наборов элементов поля при стремлении расстояния между индексирующими множествами к бесконечности. Напомним, что функция $f: \mathbb{R}^{n} \rightarrow \mathbb{R}, n \in \mathbb{N}$, назьвается липиииевой, если

$$
\operatorname{Lip}(f):=\sup _{\substack{x, y \in \mathbb{R}^{n} \\ x \neq y}} \frac{|f(x)-f(y)|}{\|x-y\|_{1}}<\infty .
$$

Здесь $\|z\|_{1}=\left|z_{1}\right|+\cdots+\left|z_{n}\right|, z \in \mathbb{R}^{n}$. Для конечного множества $Q$ пусть $|Q|-$ его мощность. Множество $\mathbb{Z}^{d}$ снабдим метрикой

$$
\rho\left(t, t^{\prime}\right)=\max _{i=1, \ldots, d}\left|t_{i}-t_{i}^{\prime}\right|, \quad t, t^{\prime} \in \mathbb{Z}^{d} .
$$

Соответствующее расстояние между подмножествами $I, J \subset \mathbb{Z}^{d}$

$$
\operatorname{dist}(I, J)=\inf \{\rho(i, j): i \in I, j \in J\} .
$$

Работа выполнена при частичной поддержке Российского фонда фундаментальных исследований, грант № 03-01-00724. 
Нам потребуются также следующие обозначения: для $n \in \mathbb{N}$ и $\tau \in \mathbb{R}$ положим

$$
B(n, \tau)= \begin{cases}n^{\tau}, & \tau>0 \\ \ln (n \vee e), & \tau=0 \\ 1, & \tau<0\end{cases}
$$

Для $x, y \in \mathbb{R}$, как обычно, $x \vee y=\max \{x, y\}, x \wedge y=\min \{x, y\}$.

Пусть $X=\left\{X_{j}, j \in \mathbb{Z}^{d}\right\}$ - центрированное случайное поле с действительньпи значениями, заданное на вероятностном пространстве $(\Omega, \mathscr{F}, \mathrm{P})$. Для $p \geqslant 1$ положим

$$
D_{p}=\sup _{j \in \mathbb{Z}^{d}} \mathrm{E}\left|X_{j}\right|^{p}
$$

Для $W \subset \mathbb{Z}^{d},|W|<\infty$, пусть $S_{W}=\sum_{j \in W} X_{j}$.

ОПРедЕлЕниЕ. Поле $X$ называется $(B L, \theta)$-зависимым, если существует последовательность $\theta_{r} \downarrow 0, r \in \mathbb{N}$, такая, что для любых конечньх непересекающихся множеств $I, J \subset \mathbb{Z}^{d}$ таких, что $\operatorname{dist}(I, J) \geqslant r$, где $r \in \mathbb{N}$, и ограниченных липшицевых функций $F: \mathbb{R}^{|I|} \rightarrow \mathbb{R}$ и $G: \mathbb{R}^{|J|} \rightarrow \mathbb{R}$ справедливо неравенство

$$
\left|\operatorname{cov}\left(F\left(X_{i}, i \in I\right), G\left(X_{j}, j \in J\right)\right)\right| \leqslant \operatorname{Lip}(F) \operatorname{Lip}(G)(|I|+|J|) \theta_{r} .
$$

Имеются интересные модели случайных полей, обладающих свойством (1) и некоторыми сходными свойствами, где $\theta_{r}$ оценивается в терминах ковариационной функции поля $X$ (см., например, [9], [11], [12]). Будем предполагать, что существуют $c_{0}>0$ и $\lambda>0$ такие, что

$$
\theta_{r} \leqslant c_{0} r^{-\lambda}, \quad r \in \mathbb{N} \text {. }
$$

Пусть $U$ - произвольный целочисленньй параллелепипед в $\mathbb{Z}^{d}$ с длинами ребер $l_{1}, \ldots$, $l_{d} \in \mathbb{N}$, т.е. $U=V \cap \mathbb{Z}^{d}$, где $V=\left(a_{1}, a_{1}+l_{1}\right] \times \cdots \times\left(a_{d}, a_{d}+l_{d}\right]$ и $a_{1}, \ldots, a_{d} \in \mathbb{Z}$. Обозначим $\mathscr{U}$ класс таких множеств $U$.

ТеОрема 1. Пусть $X=\left\{X_{j}, j \in \mathbb{Z}^{d}\right\}$ является $(B L, \theta)$-зависимым иентрированным случайным полем, удовлетворяющим условию (2). Предположим, что $D_{p}<\infty$ для некоторого $p>4$. Тогда для любого $U \in \mathscr{U}$ справедлива оченка

$$
\mathrm{E} S_{U}^{4} \leqslant|U|\left(D_{4}+6 D_{2}^{2}|U|+C_{1}(d, \tau, v) c_{0}^{v} D_{p}^{2 /(p-2)} B(|U|, \tau)\right)
$$

əде $c_{0}$ фигурирует в (2),

$$
\begin{array}{cc}
v=\frac{p-4}{p-2}, & \tau=3-\frac{\lambda v}{d}, \quad C_{1}(d, \tau, v)=290 d^{2} 3^{3 d} v^{-1} \psi(\tau), \\
\psi(\tau)= \begin{cases}\tau^{-1} \vee 1, & \tau>0 \\
2, & \tau=0, \\
2\left(|\tau|^{-1} \vee 1\right), & \tau<0 .\end{cases}
\end{array}
$$


ДокАЗАТЕЛЬСТво. Имеем

$$
\begin{aligned}
\mathrm{E} S_{U}^{4} & =\sum_{j \in U} \mathrm{E} X_{j}^{4}+\sum_{J_{4} \in \Lambda} \mathrm{E} X_{j_{1}} X_{j_{2}} X_{j_{3}} X_{j_{4}}+3 \sum_{\substack{j_{1}, j_{2} \in U \\
j_{1} \neq j_{2}}} \mathrm{E} X_{j_{1}}^{2} X_{j_{2}}^{2} \\
& =S^{(1)}+S^{(2)}+3 S^{(3)} ;
\end{aligned}
$$

здесь вторая сумма берется по множеству $\Lambda$ всех упорядоченных наборов индексов $J_{4}=$ $\left(j_{1}, j_{2}, j_{3}, j_{4}\right)$ таких, что хотя бы один из четырех индексов отличен от каждого из других.

Очевидно, $S^{(1)} \leqslant D_{4}|U|$. Для оценки $S^{(2)}$ представим множество четверок индексов, по которьм ведется суммирование в $S^{(2)}$, в виде объединения множеств $\Lambda_{r}, r=1, \ldots, l$, по следующему правилу: набор $T_{4}=\left(t_{1}, t_{2}, t_{3}, t_{4}\right)$ принадлежит типу $\Lambda_{r}$, если

$$
\max _{q=1,2,3,4} \operatorname{dist}\left(\left\{t_{q}\right\}, T_{4} \backslash\left\{t_{q}\right\}\right)=r .
$$

Без ограничения общности можно считать, что длины ребер $U$ связаны неравенствами $l_{1} \leqslant \cdots \leqslant l_{d}$. Пусть $l_{0}=0$. Тогда каждому $r=1, \ldots, l_{d}$ однозначно сопоставляется $k(r), 0 \leqslant k \leqslant d-1$, такое, что $l_{k}<r \leqslant l_{k+1}$. Нетрудно показать (аналогично [9], [13]), что

$$
\left|\Lambda_{r}\right| \leqslant 144 d\left(\prod_{i=1}^{k} l_{i}\right)^{3}(3 r)^{3(d-k)-1}|U|,
$$

где $k=k(r)$ (как обычно, произведение по пустому множеству индексов считается равным единице). Зафиксируем набор $J_{4}=\left(j_{1}, j_{2}, j_{3}, j_{4}\right) \in \Lambda_{r}, r=1, \ldots, l$. Не ограничивая обшности, можно считать, что $\operatorname{dist}\left(\left\{j_{1}\right\}, T_{4} \backslash\left\{j_{1}\right\}\right)=r$. Для упрощения записи далее будем писать $X_{q}$ вместо $X_{j_{q}}, q=1,2,3,4$. Очевидно, Е $X_{1} X_{2} X_{3} X_{4}=\operatorname{cov}\left(X_{1}, X_{2} X_{3} X_{4}\right)$.

Пусть $A>0$. Введем функцию

$$
H_{A}(t)=(|t| \wedge A) \cdot \operatorname{sgn}(t), \quad t \in \mathbb{R}
$$

Тогда $\left|H^{A}(t)\right| \leqslant A, t \in \mathbb{R}$, и $\operatorname{Lip}\left(H_{A}\right)=1$. Для $u=2,3,4$ представим случайные величины $X_{u}$ в виде $X_{u}=X_{u}^{\prime}+X_{u}^{\prime \prime}$, где $X_{u}^{\prime}=H_{A}\left(X_{u}\right), X_{u}^{\prime \prime}=X_{u}-X_{u}^{\prime}$. Ясно, что

$$
\begin{aligned}
\left|\operatorname{cov}\left(X_{1}, X_{2} X_{3} X_{4}\right)\right| \leqslant & \left|\operatorname{cov}\left(X_{1}, X_{2}^{\prime} X_{3}^{\prime} X_{4}^{\prime}\right)\right|+\left|\operatorname{cov}\left(X_{1}, X_{2}^{\prime \prime} X_{3}^{\prime} X_{4}^{\prime}\right)\right| \\
& +\left|\operatorname{cov}\left(X_{1}, X_{2}^{\prime} X_{3}^{\prime \prime} X_{4}^{\prime}\right)\right|+\left|\operatorname{cov}\left(X_{1}, X_{2}^{\prime} X_{3}^{\prime} X_{4}^{\prime \prime}\right)\right| \\
& +\left|\operatorname{cov}\left(X_{1}, X_{2}^{\prime \prime} X_{3}^{\prime \prime} X_{4}^{\prime}\right)\right|+\left|\operatorname{cov}\left(X_{1}, X_{2}^{\prime \prime} X_{3}^{\prime} X_{4}^{\prime \prime}\right)\right| \\
& +\left|\operatorname{cov}\left(X_{1}, X_{2}^{\prime} X_{3}^{\prime \prime} X_{4}^{\prime \prime}\right)\right|+\left|\operatorname{cov}\left(X_{1}, X_{2}^{\prime \prime} X_{3}^{\prime \prime} X_{4}^{\prime \prime}\right)\right| .
\end{aligned}
$$

По определению $(B L, \theta)$-зависимости $\left|\operatorname{cov}\left(X_{1}, X_{2}^{\prime} X_{3}^{\prime} X_{4}^{\prime}\right)\right| \leqslant 4 c_{0} A^{2} r^{-\lambda}$.

Для $x>0$ положим

$$
f_{x}(t)=I\{t \geqslant x\}-I\{t \leqslant-x\}, \quad t \in \mathbb{R} .
$$

Используя просто проверяемое равенство

$$
X_{u}^{\prime \prime}=\int_{A}^{+\infty} f_{x}\left(X_{u}\right) d x, \quad u=2,3,4,
$$


и теорему Фубини, имеем

$$
\left|\operatorname{cov}\left(X_{1}, X_{2}^{\prime \prime} X_{3}^{\prime} X_{4}^{\prime}\right)\right|=\left|\operatorname{cov}\left(X_{1}, \int_{A}^{\infty} f_{x}\left(X_{2}\right) X_{3}^{\prime} X_{4}^{\prime} d x\right)\right| \leqslant \int_{A}^{\infty}\left|\mathrm{E} X_{1} f_{x}\left(X_{2}\right) X_{3}^{\prime} X_{4}^{\prime}\right| d x
$$

Теперь оценим подынтегральное выражение. Применяя неравенства Гёльдера и Чебышева, имеем

$$
\begin{aligned}
\left|\mathrm{E} X_{1} f_{x}\left(X_{2}\right) X_{3}^{\prime} X_{4}^{\prime}\right| & \leqslant \mathrm{E}\left|X_{1} f_{x}\left(X_{2}\right) X_{3}^{\prime} X_{4}^{\prime}\right| \\
& \leqslant A^{2}\left(\mathrm{E}\left|X_{1}\right|^{p}\right)^{1 / p}\left(\mathrm{P}\left(\left|X_{2}\right| \geqslant x\right)\right)^{(p-1) / p} \leqslant D_{p} A^{2} x^{1-p}
\end{aligned}
$$

т.е.

$$
\left|\operatorname{cov}\left(X_{1}, X_{2}^{\prime \prime} X_{3}^{\prime} X_{4}^{\prime}\right)\right| \leqslant D_{p} A^{2} \int_{A}^{\infty} x^{1-p} d x=\frac{D_{p}}{p-2} A^{4-p} .
$$

Аналогично оцениваются $\left|\operatorname{cov}\left(X_{1}, X_{2}^{\prime} X_{3}^{\prime \prime} X_{4}^{\prime}\right)\right|$ и $\left|\operatorname{cov}\left(X_{1}, X_{2}^{\prime} X_{3}^{\prime} X_{4}^{\prime \prime}\right)\right|$. Для слагаемого $\left|\operatorname{cov}\left(X_{1}, X_{2}^{\prime \prime} X_{3}^{\prime \prime} X_{4}^{\prime}\right)\right|$, пользуясь теми же рассуждениями, получим, что

$$
\left|\operatorname{cov}\left(X_{1}, X_{2}^{\prime \prime} X_{3}^{\prime \prime} X_{4}^{\prime}\right)\right| \leqslant \int_{A}^{\infty} \int_{A}^{\infty}\left|\mathrm{E} X_{1} f_{x_{2}}\left(X_{2}\right) f_{x_{3}}\left(X_{3}\right) X_{4}^{\prime}\right| d x_{2} d x_{3} .
$$

Подьнтегральное выражение оценим так же, как в $(8)$, используя то, что $\mathrm{P}\left(\left|X_{2}\right| \geqslant x_{2}\right.$, $\left.\left|X_{3}\right| \geqslant x_{3}\right) \leqslant \mathrm{P}\left(\left|X_{2}\right| \geqslant x_{2}\right) \wedge \mathrm{P}\left(\left|X_{3}\right| \geqslant x_{3}\right)$ для любых $x_{2}>0, x_{3}>0$. Подставляя полученное выражение в (9), придем к оценке

$$
\left|\operatorname{cov}\left(X_{1}, X_{2}^{\prime \prime} X_{3}^{\prime \prime} X_{4}^{\prime}\right)\right| \leqslant D_{p} A \int_{A}^{\infty} \int_{A}^{\infty}\left(x_{2}^{1-p} \wedge x_{3}^{1-p}\right) d x_{2} d x_{3} \leqslant \frac{2 D_{p}}{p-3} A^{4-p} .
$$

Аналогично оцениваются $\left|\operatorname{cov}\left(X_{1}, X_{2}^{\prime \prime} X_{3}^{\prime} X_{4}^{\prime \prime}\right)\right|$ и $\left|\operatorname{cov}\left(X_{1}, X_{2}^{\prime} X_{3}^{\prime \prime} X_{4}^{\prime \prime}\right)\right|$. Наконец, для слагаемого $\left|\operatorname{cov}\left(X_{1}, X_{2}^{\prime \prime} X_{3}^{\prime \prime} X_{4}^{\prime \prime}\right)\right|$ те же рассуждения приводят к тройному интегралу

$$
\left|\operatorname{cov}\left(X_{1}, X_{2}^{\prime \prime} X_{3}^{\prime \prime} X_{4}^{\prime \prime}\right)\right| \leqslant D_{p} \int_{A}^{\infty} \int_{A}^{\infty} \int_{A}^{\infty}\left(x_{2} \vee x_{3} \vee x_{4}\right)^{1-p} d x_{2} d x_{3} d x_{4} \leqslant \frac{3 D_{p}}{p-4} A^{4-p}
$$

Суммируя полученные оценки, видим, что

$$
\left|\operatorname{cov}\left(X_{1}, X_{2} X_{3} X_{4}\right)\right| \leqslant 4 c_{0} A^{2} r^{-\lambda}+\frac{12 D_{p}}{p-4} A^{4-p} .
$$

Оптимизация по $A$ показывает, что

$$
\left|\operatorname{cov}\left(X_{1}, X_{2} X_{3} X_{4}\right)\right| \leqslant 4 v^{-1} c_{0}^{v}\left(\frac{3 D_{p}}{2}\right)^{2 /(p-2)} r^{-\lambda v}
$$

где $v$ введено в (4). Таким образом, учитывая (5), имеем

$$
\begin{aligned}
\left|S^{(2)}\right| & \leqslant 4 v^{-1} c_{0}^{v}\left(\frac{3 D_{p}}{2}\right)^{2 /(p-2)} \sum_{r=1}^{l_{d}}\left|\Lambda_{r}\right| r^{-\lambda v} \\
& \leqslant 576 d|U| v^{-1} c_{0}^{v}\left(\frac{3 D_{p}}{2}\right)^{\frac{2}{p-2}} \sum_{k=0}^{d-1}\left(\prod_{i=1}^{k} l_{i}\right)^{3} \sum_{r: k(r)=k} 3^{3(d-k)-1} r^{3(d-k)-1-\lambda v} \\
& \leqslant 288 d|U| 3^{3 d} v^{-1} c_{0}^{v} D_{p}^{2 /(p-2)} \sum_{k=0}^{d-1}\left(\prod_{i=1}^{k} l_{i}\right)^{\tau} \sum_{r=l_{k}+1}^{l_{k+1}} r^{(d-k) \tau-1}
\end{aligned}
$$


где $\tau$ введено в (4), а сумма по пустому множеству индексов считается равной нулю. Отсюда нетрудно получить, что

$$
\left|S^{(2)}\right| \leqslant 288 d^{2} \cdot 3^{3 d} v^{-1} c_{0}^{v} D_{p}^{2 /(p-2)} \psi(\tau)|U| B(|U|, \tau) .
$$

Обратимся теперь к оценке $S^{(3)}$. Воспользуемся представлением

$$
X_{u}^{2}=Z_{u}^{\prime}+Z_{u}^{\prime \prime}, \quad Z_{u}^{\prime}=H_{A^{2}}\left(X_{u}^{2}\right),
$$

где $A>0$ и $u=j_{1}, j_{2}$. Тогда

$$
\mathrm{E} X_{j_{1}}^{2} X_{j_{2}}^{2} \leqslant \mathrm{E} Z_{j_{1}}^{\prime} \mathrm{E} Z_{j_{2}}^{\prime}+\left|\operatorname{cov}\left(Z_{j_{1}}^{\prime}, Z_{j_{2}}^{\prime}\right)\right|+\mathrm{E} Z_{j_{1}}^{\prime} Z_{j_{2}}^{\prime \prime}+\mathrm{E} Z_{j_{1}}^{\prime \prime} Z_{j_{2}}^{\prime}+\mathrm{E} Z_{j_{1}}^{\prime \prime} Z_{j_{2}}^{\prime \prime}
$$

Очевидно, $\mathrm{E} Z_{j_{1}}^{\prime} \mathrm{E} Z_{j_{2}}^{\prime} \leqslant D_{2}^{2}$; далее, учитьвая, что

$$
Z_{u}^{\prime \prime}=2 \int_{A}^{\infty} x I\left\{\left|X_{u}\right| \geqslant x\right\} d x
$$

аналогично (7)-(10) получаем, что для $j_{1}, j_{2} \in U$ таких, что $\left|j_{1}-j_{2}\right|=r, r=1, \ldots, l$,

$$
\mathrm{E} X_{j_{1}}^{2} X_{j_{2}}^{2} \leqslant D_{2}^{2}+8 v^{-1} c_{0}^{v} D_{p}^{2 /(p-2)} r^{-\lambda v}
$$

Суммируя по $r$ и сравнивая каждое слагаемое с соответствующими элементами суммы в (11), получаем утверждение теоремы.

ЗАмечаниЕ 1. С помощью неравенства Коши-Буняковского-Шварца имеем $\left|S^{(3)}\right| \leqslant$ $D_{4}|U|^{2}$. Оценка $\mathrm{E} S_{U}^{4} \leqslant C|U|^{2}$, где $C$ не зависит от $U$, может быть также получена с помощью результата работы [14]. Однако в доказанной теореме коэффициент при $|U|^{2}$ в правой части (3) не зависит от моментов величин $X_{j}$ порядка вьше, чем 2 . Это будет использовано при доказательстве теоремы 3.

Далее потребуются новые обозначения. Назовем целочисленный параллелепипед $V \subseteq U$, где $U \in \mathscr{U}$, стандартно вложенным в $U$, если минимальная вершина $V$ (в смысле лексикографического порядка в $\mathbb{Z}^{d}$ ) совпадает с минимальной вершиной $U$, и обозначим это соотношение $V \triangleleft U$. Пусть

$$
M_{U}=\max _{V \triangleleft U}\left|S_{V}\right|
$$

Задача оценки максимума модуля сумм, берущихся по произвольным целочисленньп параллелепипедам, содержащимся в $U$, сводится к оценке $M_{U}$ (см., например, [3]).

СлЕДСТВИЕ 1. Пусть выполнены условия предыдущей теоремы $u \lambda \geqslant 2 d v^{-1}$. Тогда

$$
\mathrm{E} M_{U}^{4} \leqslant \alpha^{d}\left(7 D_{4}+C_{1}(d, \tau, v) c_{0}^{v} D_{p}^{2 /(p-2)}\right)|U|^{2}
$$

əде $\alpha=(5 / 2)\left(1-2^{-1 / 4}\right)^{-4}$.

ДокАЗАТЕЛЬСтво. Это утверждение следует из (3) и известной теоремы Морица (см. [15, следствие 1]). 
Теорема 2. Пусть $X$ является $(B L, \theta)$-зависимым иентрированным случайныцм полем, причем $D_{2}<\infty$ и выполнено (2). Тогда для произвольного $m \in \mathbb{N} u$ любого челочисленного параллелепипеда $U$ верна оченка

$$
\mathrm{E} M_{U}^{2} \leqslant 3 m^{d}\left(D_{1}^{2}|U|^{2}+18 \sum_{j \in U} \mathrm{E} X_{j}^{2}+16 c_{0}|U| m^{-\lambda}\right)
$$

ДокАЗАТЕЛЬСтво. Не ограничивая общности, можно считать, что вероятностное пространство $(\Omega, \mathscr{F}, \mathrm{P})=\left(\Omega_{1}, \mathscr{F}_{1}, \mathrm{P}_{1}\right) \otimes\left(\Omega_{2}, \mathscr{F}_{2}, \mathrm{P}_{2}\right)$, причем случайные величины $\left\{X_{j}\left(\omega_{1}\right), j \in U, \omega_{1} \in \Omega_{1}\right\}$ заданы на $\left(\Omega_{1}, \mathscr{F}_{1}, \mathrm{P}_{1}\right)$ и на $\left(\Omega_{2}, \mathscr{F}_{2}, \mathrm{P}_{2}\right)$ существует случайное поле $\left\{\varepsilon_{j}\left(\omega_{2}\right), j \in \mathbb{Z}^{d}, \omega_{2} \in \Omega_{2}\right\}$, где величины $\varepsilon_{i}$ независимы и каждая из них принимает значения 1 и -1 с вероятностью $1 / 2$. Для случайных величин $Y\left(\omega_{1}, \omega_{2}\right)=F\left(X_{j}\left(\omega_{1}\right), \varepsilon_{j}\left(\omega_{2}\right), j \in U\right), Y^{\prime}\left(\omega_{1}, \omega_{2}\right)=G\left(X_{j}\left(\omega_{1}\right), \varepsilon_{j}\left(\omega_{2}\right), j \in U\right)$, где $F: \mathbb{R}^{2|U|} \rightarrow \mathbb{R}$ и $G: \mathbb{R}^{2|U|} \rightarrow \mathbb{R}$ - борелевские функции, будем обозначать

$$
\begin{gathered}
\mathrm{E}_{1} Y=\int_{\Omega_{1}} Y d \mathrm{P}_{1}, \quad \mathrm{E}_{2} Y=\int_{\Omega_{2}} Y d \mathrm{P}_{2}, \\
\operatorname{cov}_{1}\left(Y, Y^{\prime}\right)=\mathrm{E}_{1} Y Y^{\prime}-\mathrm{E}_{1} Y \mathrm{E}_{1} Y^{\prime}, \quad\|Y\|=\left(\mathrm{E}_{1} Y^{2}\right)^{1 / 2} .
\end{gathered}
$$

Очевидно, имеем $\mathrm{E}_{1} \mathrm{E}_{2} Y=\mathrm{E}_{2} \mathrm{E}_{1} Y=\mathrm{E} Y$, а если при каждом $\omega_{2} \in \Omega_{2} F$ и $G$-липшицевы функции, то для оценки ковариации $\operatorname{cov}_{1}\left(Y, Y^{\prime}\right)$ можно использовать условие зависимости (1).

Пусть $e_{0} \in U$ - минимальный элемент $U$ в смысле лексикографического порядка. Рассмотрим множество $\Gamma=\{0, \ldots, m-1\}^{d}$. Для $\gamma \in \Gamma$ введем целочисленную решетку

$$
\mathbb{Z}^{d}(\gamma)=\left\{j \in \mathbb{Z}^{d}: j=e_{0}+\left(m z_{1}, \ldots, m z_{d}\right), z_{i} \in \mathbb{Z}, i=1, \ldots, d\right\}
$$

и положим $U(\gamma)=U \cap \mathbb{Z}^{d}(\gamma)$. Очевидно,

$$
\sum_{\gamma \in \Gamma}|U(\gamma)|=|U| \text { и } \min _{\substack{i, j \in U(\gamma) \\ i \neq j}} \operatorname{dist}(i, j)=m, \quad U(\gamma) \neq \varnothing .
$$

Пусть

$$
M_{U}(\gamma)=\max _{V \triangleleft U}\left|S_{V \cap U(\gamma)}\right|
$$

Тогда

$$
\mathrm{E} M_{U}^{2} \leqslant m^{d} \sum_{\gamma \in \Gamma} \mathrm{E} M_{U}^{2}(\gamma)
$$

Оценим $\mathrm{E} M_{U}^{2}(\gamma)$ для фиксированного $\gamma \in \Gamma$ такого, что $U(\gamma) \neq \varnothing$. Следуя [5], для $V \triangleleft U$ положим

$$
\begin{gathered}
S_{1, V}=\sum_{\substack{j \in V \cap U(\gamma) \\
\varepsilon_{j}=1}} X_{j}, \quad S_{-1, V}=\sum_{\substack{j \in V \cap U(\gamma) \\
\varepsilon_{j}=-1}} X_{j}, \widetilde{S}_{V}=S_{1, V}-S_{-1, V}, \\
M=M_{U}(\gamma), \quad M_{1}=\max _{V \triangleleft U}\left|S_{1, V}\right|, \quad M_{-1}=\max _{V \triangleleft U}\left|S_{-1, V}\right|, \quad \widetilde{M}=\max _{V \triangleleft U}\left|\widetilde{S}_{V}\right| .
\end{gathered}
$$


Имеем

$$
2 M_{ \pm 1} \leqslant \max _{V \triangleleft U}\left|S_{1, V}+S_{-1, V}\right|+\max _{V \triangleleft U}\left|S_{1, V}-S_{-1, V}\right| \leqslant M+\widetilde{M}
$$

следовательно, $M_{1}+M_{-1} \leqslant M+\widetilde{M}$. Очевидно,

$$
M \leqslant M_{1}+M_{-1}, \quad\left|M_{1}-M_{-1}\right| \leqslant \widetilde{M}
$$

Так как $M$ не зависит от $\left\{\varepsilon_{j}, j \in U\right\}$, то $\left(\mathrm{E} M^{2}\right)^{1 / 2}=\|M\| \leqslant\left\|M_{1}\right\|+\left\|M_{-1}\right\|$. Пусть

$$
\xi=\frac{M_{1}}{\left\|M_{1}\right\| \vee \beta}, \quad \eta=\frac{M_{-1}}{\left\|M_{-1}\right\| \vee \beta},
$$

где $\beta=\left(c_{0}|U(\gamma)| m^{-\lambda}\right)^{1 / 2}$, причем в случае, когда знаменатель какой-либо из дробей равен нулю, вся дробь полагается равной нулю. Легко видеть, что $\|\xi\| \leqslant 1,\|\eta\| \leqslant 1$. При каждом $\omega_{2} \in \Omega_{2}$ множества $\left\{j \in U(\gamma): \varepsilon_{j}=1\right\}$ и $\left\{j \in U(\gamma): \varepsilon_{j}=-1\right\}$ не пересекаются, и величины $M_{1}$ и $M_{-1}$ представляют собой липшицевы функции от непересекающихся наборов величин $X_{j}, j \in U(\gamma)$. Для этих функций константы Липшица равны 1. Поэтому

$$
\begin{aligned}
\mathrm{E}_{1} \xi M_{-1} & =\operatorname{cov}_{1}\left(\xi, M_{-1}\right)+\mathrm{E}_{1} \xi \mathrm{E}_{1} M_{-1} \\
& \leqslant\left|\operatorname{cov}_{1}\left(\xi, M_{-1}\right)\right|+\|\xi\| \mathrm{E}_{1} M_{-1} \leqslant \beta+\mathrm{E}_{1} M_{-1}
\end{aligned}
$$

ввиду $(B L, \theta)$-зависимости и (13). Аналогично $\mathrm{E}_{1} \eta M_{1} \leqslant \beta+\mathrm{E}_{1} M_{1}$. Заметим также, что если $\left\|M_{1}\right\| \geqslant \beta$, то $\mathrm{E}_{1} \xi M_{1}=\left\|M_{1}\right\|$, а если $\left\|M_{-1}\right\| \geqslant \beta$, то $\mathrm{E}_{1} \eta M_{-1}=\left\|M_{-1}\right\|$.

Разберем четыре случая.

Случай 1: $\left\|M_{1}\right\| \geqslant \beta,\left\|M_{-1}\right\| \geqslant \beta$. В силу доказанных равенств и оценок

$$
\begin{aligned}
\left(\mathrm{E}^{2}\right)^{1 / 2} & \leqslant\left\|M_{1}\right\|+\left\|M_{-1}\right\|=\mathrm{E}_{1} \xi M_{1}+\mathrm{E}_{1} \eta M_{-1} \\
& =\mathrm{E}_{1}(\xi-\eta)\left(M_{1}-M_{-1}\right)+\mathrm{E}_{1} \xi M_{-1}+\mathrm{E}_{1} \eta M_{1} \\
& \leqslant\|\xi-\eta\|\left\|M_{1}-M_{-1}\right\|+2 \beta+\mathrm{E}_{1}\left(M_{1}+M_{-1}\right) \leqslant 2\|\widetilde{M}\|+2 \beta+\mathrm{E}_{1}(M+\widetilde{M}) \\
& \leqslant 3\|\widetilde{M}\|+2 \beta+\mathrm{E}_{1} M .
\end{aligned}
$$

Случай 2: $\left\|M_{1}\right\| \geqslant \beta,\left\|M_{-1}\right\|<\beta$. Тогда $\mathrm{E}_{1} \eta M_{-1} \leqslant \beta$. Поэтому

$$
\begin{aligned}
\left(\mathrm{E}^{2}\right)^{1 / 2} & \leqslant\left\|M_{1}\right\|+\left\|M_{-1}\right\| \leqslant \mathrm{E}_{1} \xi M_{1}+\beta+\mathrm{E}_{1} \eta M_{-1}-\mathrm{E}_{1} \eta M_{-1} \\
& \leqslant\left|\beta-\mathrm{E}_{1} \eta M_{-1}\right|+\mathrm{E}_{1} \xi M_{1}+\mathrm{E}_{1} \eta M_{-1} \leqslant 2 \beta+\mathrm{E}_{1} \xi M_{1}+\mathrm{E}_{1} \eta M_{-1} \\
& \leqslant 3\|\widetilde{M}\|+4 \beta+\mathrm{E}_{1} M
\end{aligned}
$$

аналогично первому случаю.

Случай 3: $\left\|M_{1}\right\|<\beta,\left\|M_{-1}\right\| \geqslant \beta$. Рассматривается аналогично случаю 2.

Случай 4: $\left\|M_{1}\right\|<\beta,\left\|M_{-1}\right\|<\beta$. В этом случае

$$
\left(\mathrm{E} M^{2}\right)^{1 / 2} \leqslant\left\|M_{1}\right\|+\left\|M_{-1}\right\|<2 \beta .
$$

Итак, имеем $\left(\mathrm{E} M^{2}\right)^{1 / 2} \leqslant 3\|\widetilde{M}\|+4 \beta+\mathrm{E} M$. Таким образом,

$$
\mathrm{E} M^{2} \leqslant 3\left(9 \mathrm{E} \widetilde{M}^{2}+(\mathrm{E} M)^{2}+16 c_{0}|U(\gamma)| m^{-\lambda}\right)
$$


Оценим Е $\widetilde{M^{2}}$. При фиксированном $\omega_{1} \in \Omega_{1}$

$$
\widetilde{M}=\max _{V \triangleleft U}\left|\sum_{j \in V \cap U(\gamma)} \zeta_{j}\right|
$$

где $\zeta_{j}=\varepsilon_{j} X_{j}$ - независимые симметричные случайные величины (на вероятностном пространстве $\left.\left(\Omega_{2}, \mathscr{F}_{2}, \mathrm{P}_{2}\right)\right)$ с $\mathrm{E}_{2} \zeta_{j}=0$ и $\mathrm{E}_{2} \zeta_{j}^{2}=X_{j}^{2}, j \in U$. Применяя принцип отражения, видим, что

$$
\mathrm{E}_{2} \widetilde{M}^{2} \leqslant 2 \mathrm{E}_{2}\left(\sum_{j \in U(\gamma)} \zeta_{j}\right)^{2} \leqslant 2 \sum_{j \in U(\gamma)} X_{j}^{2}
$$

поэтому

$$
\mathrm{E} \widetilde{M^{2}}=\mathrm{E}_{1} \mathrm{E}_{2} \widetilde{M}^{2} \leqslant 2 \sum_{j \in U(\gamma)} \mathrm{E} X_{j}^{2}
$$

Принимая во внимание (13)-(16), получаем, что

$$
\begin{aligned}
\mathrm{E} M_{U}^{2} & \leqslant 3 m^{d} \sum_{\gamma \in \Gamma}\left(18 \sum_{j \in U(\gamma)} \mathrm{E} X_{j}^{2}+\left(\sum_{j \in U(\gamma)} \mathrm{E}\left|X_{j}\right|\right)^{2}+16 c_{0}|U(\gamma)| m^{-\lambda}\right) \\
& \leqslant 3 m^{d}\left(18 \sum_{j \in U} \mathrm{E} X_{j}^{2}+D_{1}^{2} \sum_{\gamma \in \Gamma}|U(\gamma)|^{2}+16 c_{0}|U| m^{-\lambda}\right) \\
& \leqslant 3 m^{d}\left(18 \sum_{j \in U} \mathrm{E} X_{j}^{2}+D_{1}^{2}|U|^{2}+16 c_{0}|U| m^{-\lambda}\right) .
\end{aligned}
$$

Теорема доказана.

ТЕОрема 3. Пусть $X$ есть $(B L, \theta)$-зависимое чентрированное случайное поле, и существует $q \geqslant 2$ такое, что $D_{q}<\infty$, а $\lambda$ в (2) удовлетворяет условию $\lambda>3 d$. Тогда для любого $U \in \mathscr{U}$ справедливо неравенство

$$
|U|^{-1} \mathrm{E} M_{U}^{2} \leqslant \Delta=\left(96 c_{0}+C_{2}(d, \lambda)\left(\alpha^{d} c_{0}\right)^{1 / 2}+\left(2\left(6 \alpha^{d}\right)^{1 / 2}+432\right) D_{2}+24 D_{2}^{2}+8\left(\alpha^{d} D_{q}\right)^{1 / 2}\right),
$$

где а введено в $(12), C_{2}(d, \lambda)=97 d \cdot 3^{3 d / 2}\left(\left(\lambda d^{-1}-3\right)^{-1 / 2} \vee 1\right)$. Если, кроме того, случайное поле $\left\{X_{j}^{2}, j \in \mathbb{Z}^{d}\right\}$ равномерно интегрируемо, то и семейство случайных величин $\left\{|U|^{-1} M_{U}^{2}, U \in \mathscr{U}\right\}$ равномерно интегрируемо.

ДокАЗАТЕльСтво. Введем центрированные урезанные случайные величины

$$
X_{j}^{\prime}=H_{\sqrt{|U|}}\left(X_{j}\right)-\mathrm{E} H_{\sqrt{|U|}}\left(X_{j}\right), \quad X_{j}^{\prime \prime}=X_{j}-X_{j}^{\prime}, \quad j \in U
$$

где функция $H_{A}$ определена согласно формуле (6). Положим

$$
S_{U}^{\prime}=\sum_{j \in V} X_{j}^{\prime}, \quad S_{U}^{\prime \prime}=\sum_{j \in V} X_{j}^{\prime \prime}, \quad M_{U}^{\prime}=\max _{V \triangleleft U}\left|S_{V}^{\prime}\right|, \quad M_{U}^{\prime \prime}=\max _{V \triangleleft U}\left|S_{V}^{\prime \prime}\right| .
$$

Для оценки моментов величин $X_{j}$ и $X_{j}^{\prime \prime}$ используем простую оценку

$$
\mathrm{E}|Z-\mathrm{E} Z|^{p} \leqslant 2^{p} \mathrm{E}|Z|^{p},
$$


справедливую для любого $p \geqslant 1$ и любой случайной величины $Z$, имеющей момент порядка $p$.

Пусть $m \in \mathbb{N}$ - число, значение которого будет подобрано позже. Оценим $\mathrm{E}\left(M_{U}^{\prime \prime}\right)^{2}$ c помощью (17) и теоремы 2 (аналогично [5]):

$$
\begin{aligned}
\mathrm{E}\left(M_{U}^{\prime \prime}\right)^{2} \leqslant & 12 m^{d}\left(\left(|U| \max _{j \in U} \mathrm{E}\left|X_{j}\right| I\left\{\left|X_{j}\right| \geqslant \sqrt{|U|}\right\}\right)^{2}\right. \\
& \left.+18|U| \max _{j \in U} \mathrm{E} X_{j}^{2} I\left\{\left|X_{j}\right| \geqslant \sqrt{|U|}\right\}+4 c_{0}|U| m^{-\lambda}\right) \\
\leqslant & 12 m^{d}|U|\left(b^{2}(\sqrt{|U|})+18 b(\sqrt{|U|})+4 c_{0} m^{-\lambda}\right)
\end{aligned}
$$

где

$$
b(x)=\sup _{j \in \mathbb{Z}^{d}} \mathrm{E} X_{j}^{2} I\left\{\left|X_{j}\right| \geqslant x\right\} \leqslant D_{2}, \quad x>0 .
$$

Теперь оценим $\mathrm{E} M_{U}^{\prime}{ }^{4}$, пользуясь теоремой 1 . Величины $X_{j}^{\prime}$ ограничены и, следовательно, имеют конечные абсолютные моменты всех порядков. Для каждого $p \geqslant q$, используя (17), нетрудно показать, что

$$
D_{p}^{\prime}=\sup _{j \in \mathbb{Z}^{d}} \mathrm{E}\left|X_{j}^{\prime}\right|^{p} \leqslant 2^{p}|U|^{(p-q) / 2} D_{q} .
$$

Пусть $p>4, p \geqslant q$. Применяя теорему 1, с учетом (19) получаем, что

$$
\begin{aligned}
\mathrm{E} S_{U}^{\prime}{ }^{4} \leqslant & |U|\left(6 D_{2}^{2}|U|+D_{4}^{\prime}+C_{1}(d, \tau, v) c_{0}^{v} D_{p}^{\prime 2 /(p-2)} B(|U|, \tau)\right) \\
\leqslant & |U|\left(6 D_{2}^{2}|U|+16 D_{q}|U|^{(2-q / 2) \vee 0}\right. \\
& \left.+2^{(2 p) /(p-2)} C_{1}(d, \tau, v) c_{0}^{v}|U|^{(p-q) /(p-2)} D_{q}^{2 /(p-2)} B(|U|, \tau)\right)
\end{aligned}
$$

где $v, \tau$ и $C_{1}(d, \tau, v)$ определены в $(4)$.

При всех достаточно больших $p$ имеем $\tau<0$ и, следовательно, $B(|U|, \tau)=1$. Устремив $p$ к бесконечности, приходим к оценке

$$
\mathrm{E} S_{U}^{\prime 4} \leqslant\left(6 D_{2}^{2}+16 D_{q}+2320 d^{2} \cdot 3^{3 d}\left(\left(\lambda d^{-1}-3\right)^{-1} \vee 1\right) c_{0}\right)|U|^{2}
$$

Снова используя теорему Морица, получаем, что при $\lambda>3 d$ вьполнено неравенство

$$
\mathrm{E}\left(M_{U}^{\prime}\right)^{4} \leqslant \alpha^{d} \mathrm{E}\left(S_{U}^{\prime}\right)^{4} \leqslant \alpha^{d}\left(6 D_{2}^{2}+16 D_{q}+2320 d^{2} \cdot 3^{3 d}\left(\left(\lambda d^{-1}-3\right)^{-1} \vee 1\right) c_{0}\right)|U|^{2}
$$

Чтобы доказать первое утверждение теоремы, возьмем $m=1$ в (18). Ввиду (18),(20) и того, что $M_{U} \leqslant M_{U}^{\prime}+M_{U}^{\prime \prime}$, имеем

$$
\begin{aligned}
\mathrm{E}|U|^{-1} M_{U}^{2} \leqslant & 2|U|^{-1}\left(\mathrm{E} M_{U}^{\prime 2}+\mathrm{E} M_{U}^{\prime \prime 2}\right) \\
\leqslant & 24\left(b^{2}(\sqrt{|U|})+18 b(\sqrt{|U|})+4 c_{0}\right) \\
& +\alpha^{d / 2}\left(2 \sqrt{6} D_{2}+8 D_{q}^{1 / 2}+C_{2}(d, \lambda) c_{0}^{1 / 2}\right) \leqslant \Delta .
\end{aligned}
$$

Пусть теперь семейство $\left\{X_{j}^{2}, j \in \mathbb{Z}^{d}\right\}$ равномерно интегрируемо. Тогда $b(x) \rightarrow 0$, $x \rightarrow+\infty$. Пусть $m(n), n \in \mathbb{N},-$ последовательность натуральных чисел такая, что $m(n) \rightarrow \infty$ и $m^{d}(n) b(\sqrt{n}) \rightarrow 0$ при $n \rightarrow \infty$. 
Возьмем в (18) $m=m(|U|)$. Так как $\lambda>3 d$, при таком выборе $m$ из (18) следует, что $|U|^{-1} \mathrm{E} M_{U}^{\prime \prime 2} \rightarrow 0,|U| \rightarrow \infty$. Для произвольного $\mu>0$ имеем

$$
\begin{aligned}
|U|^{-1} \mathrm{E} M_{U}^{2} I\left\{M_{U}^{2} \geqslant \mu|U|\right\} & \leqslant 6|U|^{-1} \mathrm{E}\left(M_{U}^{\prime \prime}\right)^{2}+2|U|^{-1} \mathrm{E}\left(M_{U}^{\prime}\right)^{2} I\left\{\left(M_{U}^{\prime}\right)^{2} \geqslant \frac{\mu|U|}{4}\right\} \\
& \leqslant 6|U|^{-1} \mathrm{E}\left(M_{U}^{\prime \prime}\right)^{2}+8 \mu^{-1}|U|^{-2} \mathrm{E}\left(M_{U}^{\prime}\right)^{4} \rightarrow 0
\end{aligned}
$$

при $|U| \rightarrow \infty$ и $\mu \rightarrow \infty$, откуда легко следует равномерная интегрируемость семейства $\left\{|U|^{-1} M_{U}^{2}, U \in \mathscr{U}\right\}$. Теорема доказана.

Автор признателен профессору А.В. Булинскому за постановку задачи и помощь в работе.

\section{СПИСОК ЦИТИРОВАННОЙ ЛИТЕРАТУРЫ}

[1] Петров В. В. Предельные теоремы для сумм независимых случайных величин. М.: Наука, 1987.

[2] Doukhan P. Mixing: Properties and Examples // Lecture Notes in Statistics. V. 85. Berlin: Springer, 1994.

[3] Bulinski A. V., Keane M.S. Invariance principle for associated random fields // J. of Math. Sci. 1996. V. 81. № 5. P. 2905-2911.

[4] Wichura M. J. Inequalities with applications to the weak convergence of random processes with multi-dimensional time parameters // Ann. Math. Statist. 1969. V. 40. № 2. P. 681-687.

[5] Zhang L.-X., Wen J. A weak convergence for negatively associated fields // Statist. Probab. Lett. 2001. V. 53. № 3. P. 259-267.

[6] Биллингсли П. Сходимость вероятностных мер. М.: Наука, 1977.

[7] Булинский А. В. Функциональный закон повторного логарифма для ассоциированных случайных полей // Фундамент. и прикл. матем. 1995. Т. 1. № 3. С. 623-639.

[8] Bulinski A. V., Suquet C. Normal approximation for quasi-associated random fields // Statist. Probab. Lett. 2001. V. 54. № 2. P. 215-226.

[9] Doukhan P., Lang G. Rates in the empirical central limit theorem for stationary weakly dependent random fields // Statistical Inference for Stochastic Processes. 2002. V. 5. № 2 . P. 199-228.

[10] Doukhan P., Louhichi S. A new weak dependence condition and application to moment inequalities // Stochastic Process. Appl. 1999. V. 84. № 2. P. 313-342.

[11] Булинский А. В., Шабанович Э. Асимптотическое поведение некоторых функционалов от положительно и отрицательно зависимых случайных полей // Фундамент. и прикл. матем. 1998. T. 4. № 2. C. $479-492$.

[12] Шашкин А.П. Квазиассоциированность гауссовской системы случайных векторов // УМH. 2002. Т. 57. №6. С. 199-200.

[13] Булинский А. В. Неравенства для моментов сумм ассоциированных мультииндексированных величин // Теория вероятн. и ее примен. 1993. Т. 38. № 2. С. 417-425.

[14] Бахтин Ю. Ю., Булинский А. В. Моментные неравенства для сумм зависимых мультииндексированных величин // Фундамент. и прикл. матем. 1997. Т. 3. №4. С. 1101-1108.

[15] Moricz F. A general moment inequality for the maximum of the rectangular partial sums of multiple series // Acta Math. Hung. 1983. V. 41. № 3-4. P. 337-346.

Московский государственный университет им. М.В. Ломоносова

E-mail: shashkin@mech.math.msu.su

Поступило 27.02 .2003 